\title{
EXISTENCE AND NONEXISTENCE OF GLOBAL SOLUTIONS OF DEGENERATE AND SINGULAR PARABOLIC SYSTEMS
}

\author{
GABRIELLA CARISTI
}

Received 29 November 2000

\section{Introduction}

We study the problem of the existence and nonexistence of global weak solutions of the initial value problem for systems of parabolic inequalities of the following two types:

$$
\begin{gathered}
u_{t}-|x|^{\tau_{1}} \Delta u \geq|v|^{q}, \quad(x, t) \in \mathbb{R}^{N} \times(0, \infty), \\
v_{t}-|x|^{\tau_{2}} \Delta v \geq|u|^{p}, \quad(x, t) \in \mathbb{R}^{N} \times(0, \infty), \\
u_{t}-\Delta u \geq t^{k_{1}}|x|^{-\sigma_{1}}|v|^{q}, \quad(x, t) \in \mathbb{R}^{N} \times(0, \infty), \\
v_{t}-\Delta v \geq t^{k_{2}}|x|^{-\sigma_{2}}|u|^{p}, \quad(x, t) \in \mathbb{R}^{N} \times(0, \infty),
\end{gathered}
$$

where $p, q>1$ and $u(x, 0)=u_{0}(x), v(x, 0)=v_{0}(x), x \in \mathbb{R}^{N}$. Systems like (1.1) and (1.2) will be called degenerate and singular, respectively. Several authors have addressed this problem recently: we refer the interested reader to the papers by Levine [4] and Deng and Levine [1] for a survey of the literature on this subject. In the proofs we follow the technique developed by Mitidieri and Pohozaev in $[6,7]$, which allows to prove the nonexistence of not necessarily positive solutions avoiding the use of any comparison principle through the choice of suitable text functions and careful capacitary estimates. We emphasize that in the present paper we do not assume any sign condition on the solutions, while we ask that the initial data have the following weak weighted positivity property:

$$
\liminf _{R \rightarrow \infty} \int_{B_{R}} u_{0}|x|^{-\tau_{1}} d x>0, \quad \liminf _{R \rightarrow \infty} \int_{B_{R}} v_{0}|x|^{-\tau_{2}} d x>0
$$

where $\tau_{1}=\tau_{2}=0$ in case of system (1.2). Of course, (1.3) is in particular satisfied by positive initial data.

Throughout the paper by "nonexistence of weak solution" we mean "nonexistence of nontrivial weak solutions."

Copyright $\odot 2000$ Hindawi Publishing Corporation Abstract and Applied Analysis 5:4 (2000) 265-284

2000 Mathematics Subject Classification: 35K55, 35B33

URL: http://aaa.hindawi.com/volume-5/S1085337500000385.html 
The paper is organized as follows: in Section 2, we consider systems of type (1.1), containing subcritical degeneracies, that is, we assume that $\tau_{1}<\tau_{2} \leq 2$. The main result of this section (Theorem 2.4) recovers the result obtained in [7] for the single inequality, that is, when $\tau_{1}=\tau_{2}$ and $p=q$. Moreover, Theorem 2.4 includes as a particular case the result by Escobedo and Herrero [2], which concerns the system of equations under the assumptions that $\tau_{1}=\tau_{2}=0$ and $u_{0}, v_{0} \geq 0$.

In Section 3, we deal with critical degenerate systems like (1.1), where the term "critical" means that $\tau_{1}=\tau_{2}=2$. For the single inequality $(p=q)$, it is known (see [7]) that $q^{*}$, the critical exponent for the nonexistence of global solutions, is independent of the dimension $N$. Here we show that the same fact occurs for systems like (1.1). More precisely, in Theorem 3.4 we establish that if $\tau_{1}=\tau_{2}=2$ and $p, q>1$ satisfy the following condition:

$$
\min \{q(p-2), p(q-2)\} \leq 3,
$$

then, no weak solution of (1.1) exists. In the second part of Section 3, we prove that global solutions of system (1.1) exist when $\tau_{1}=\tau_{2}=2, p, q>1$ and (1.4) does not hold and the initial data are sufficiently small. The results of this section can be summarized by saying that the curve

$$
\min \{q(p-2), p(q-2)\}=3
$$

is the critical curve for the system (2.39).

Section 4 contains a nonexistence theorem for the singular parabolic system (1.2). Fujita-type results for system (1.2) were obtained in [8] for $k_{1}=k_{2}=0$ and in [9] for $\sigma_{1}=\sigma_{2}=0$. Theorem 4.2 includes the blowup results of $[8,9]$, giving a unique nonexistence condition containing all the parameters. This answers a question posed in Deng-Levine [1].

Throughout the paper we use the following notations: for any $p>1$ we denote by $p^{\prime}$ the conjugate exponent of $p$, that is, $1 / p+1 / p^{\prime}=1$. The symbol $C$ denotes a positive constant which may vary from line to line.

We conclude this introduction with a short remark: in the course of the proofs, we frequently use the fact that if $\phi \in C_{0}^{\infty}\left(\mathbb{R}^{N} \times[0, \infty)\right)$ is a standard cut-off function and $\rho>1$, then it is always possible to select $\phi$ in order that

$$
\int_{0}^{\infty} \int_{\mathbb{R}^{N}} \frac{|D \phi|^{\rho}}{\phi^{\rho-1}} d x d t<\infty .
$$

A justification of this fact is contained for instance in [6].

\section{Systems of parabolic differential inequalities containing subcritical degeneracies}

Set $D=\mathbb{R}^{N} \times(0,+\infty)$. We consider the following initial value problem:

$$
\begin{gathered}
u_{t}-|x|^{\tau_{1}} \Delta u \geq|v|^{q}, \quad v_{t}-|x|^{\tau_{2}} \Delta v \geq|u|^{p}, \quad(x, t) \in D, \\
u(x, 0)=u_{0}(x), \quad v(x, 0)=v_{0}(x), \quad x \in \mathbb{R}^{N},
\end{gathered}
$$

where we assume that $\tau_{1}<\tau_{2} \leq 2, p, q>1$ and $v_{0}|x|^{-\tau_{2}}, u_{0}|x|^{-\tau_{1}} \in L_{\text {loc }}^{1}\left(\mathbb{R}^{N}\right)$. 
Definition 2.1. We say that $(u, v)$ is a weak solution of (2.1) if the following assumptions are satisfied:

(i) $u, v: D \rightarrow \mathbb{R}$,

(ii) $|v|^{q}|x|^{-\tau_{1}},|u|^{p}|x|^{-\tau_{2}} \in L_{\text {loc }}^{1}(D)$,

(iii) $v|x|^{-\tau_{2}}, u|x|^{-\tau_{1}} \in L_{\mathrm{loc}}^{1}(D)$,

(iv) $u, v \in L_{\text {loc }}^{1}(D)$,

and for any nonnegative $\phi \in C_{0}^{\infty}\left(\mathbb{R}^{N} \times[0, \infty)\right)$ the following inequalities hold:

$$
\begin{aligned}
& \int_{D}|v|^{q}|x|^{-\tau_{1}} \phi d x d t \leq-\int_{D} u\left(\Delta \phi+|x|^{-\tau_{1}} \phi_{t}\right) d x d t-\int_{\mathbb{R}^{N}} u_{0}|x|^{-\tau_{1}} \phi(x, 0) d x, \\
& \int_{D}|u|^{p}|x|^{-\tau_{2}} \phi d x d t \leq-\int_{D} v\left(\Delta \phi+|x|^{-\tau_{2}} \phi_{t}\right) d x d t-\int_{\mathbb{R}^{N}} v_{0}|x|^{-\tau_{2}} \phi(x, 0) d x .
\end{aligned}
$$

In this section, we study the nonexistence of weak solutions of problem (2.1). To this aim we use the approach developed by Mitidieri and Pohozaev in [6] in the context of elliptic problems and successively modified in [5, 7] to deal with parabolic and hyperbolic problems. This technique consists in deriving careful estimates of weighted $L^{p}$-norms of solutions by choosing suitable cut-off functions and rescaling arguments.

In order to formulate our results we introduce some notations.

Let $\gamma>0$ and $R>0$ be given. For any $\phi_{0} \in C_{0}^{\infty}(\mathbb{R})$ such that $0 \leq \phi_{0}(s) \leq 1$, for any $s \in \mathbb{R}$ and

$$
\phi_{0}(s)= \begin{cases}1, & 0 \leq s \leq 1 \\ 0, & s \geq 2\end{cases}
$$

we define

$$
\phi_{\gamma}(x)=\phi_{0}\left(\frac{t}{R^{\gamma}}+\frac{|x|^{2}}{R^{2}}\right) .
$$

For any $u, v: D \rightarrow \mathbb{R}$ such that $|v|^{q}|x|^{-\tau_{1}}$ and $|u|^{p}|x|^{-\tau_{2}} \in L_{\text {loc }}^{1}(D)$, we set

$$
\Phi_{1}=\int_{D} \frac{|v|^{q}}{|x|^{\tau_{1}}} \phi_{\gamma} d x d t, \quad I_{2}=\int_{D} \frac{|u|^{p}}{|x|^{\tau_{2}}} \phi_{\gamma} d x d t .
$$

Further, given $\gamma>0$ let

$$
\begin{aligned}
& \alpha_{1}=-\gamma-\left(\tau_{1}-\frac{\tau_{2}}{p}\right)+\frac{\gamma+N}{p^{\prime}}, \\
& \alpha_{2}=-2+\frac{\tau_{2}}{p}+\frac{\gamma+N}{p^{\prime}}, \\
& \alpha_{3}=-\gamma-\left(\tau_{2}-\frac{\tau_{1}}{q}\right)+\frac{\gamma+N}{q^{\prime}}, \\
& \alpha_{4}=-2+\frac{\tau_{1}}{q}+\frac{\gamma+N}{q^{\prime}} .
\end{aligned}
$$


268 Existence and nonexistence of global solutions

Proposition 2.2. Assume that $v_{0}|x|^{-\tau_{2}}, u_{0}|x|^{-\tau_{1}} \in L_{\text {loc }}^{1}\left(\mathbb{R}^{N}\right)$ and that $(u, v)$ is a weak solution of (2.1). Then, for any $\phi_{\gamma} \in C_{0}^{\infty}\left(\mathbb{R}^{N} \times[0, \infty)\right)$ given by (2.4) the following estimates hold:

$$
\begin{aligned}
& \Phi_{1} \leq C\left(R^{\alpha_{1}}+R^{\alpha_{2}}\right) \oiint_{2}^{1 / p}-\int_{\mathbb{R}^{N}} u_{0}|x|^{-\tau_{1}} \phi_{\gamma}(x, 0) d x, \\
& \Phi_{2} \leq C\left(R^{\alpha_{3}}+R^{\alpha_{4}}\right) \mathscr{\Phi}_{1}^{1 / q}-\int_{\mathbb{R}^{N}} v_{0}|x|^{-\tau_{2}} \phi_{\gamma}(x, 0) d x .
\end{aligned}
$$

Proof. Let $(u, v)$ be a weak solution of (2.1). Applying Hölder inequality to the righthand sides of (2.2) with $\phi=\phi_{\gamma}$, we obtain

$$
\begin{aligned}
\Phi_{1} \leq & {\left[\left(\int_{D}\left|\left(\phi_{\gamma}\right)_{t}\right|^{p^{\prime}}|x|^{-\left(\tau_{1}-\tau_{2} / p\right) p^{\prime}} \phi_{\gamma}^{1-p^{\prime}} d x d t\right)^{1 / p^{\prime}}\right.} \\
& \left.+\left(\int_{D}\left|\Delta \phi_{\gamma}\right|^{p^{\prime}}|x|^{\tau_{2}\left(p^{\prime}-1\right)} \phi_{\gamma}^{1-p^{\prime}} d x d t\right)^{1 / p^{\prime}}\right] \Im_{2}^{1 / p}-\int_{\mathbb{R}^{N}} u_{0}|x|^{-\tau_{1}} \phi_{\gamma}(x, 0) d x, \\
\Phi_{2} \leq & {\left[\left(\int_{D}\left|\left(\phi_{\gamma}\right)_{t}\right|^{q^{\prime}}|x|^{-\left(\tau_{2}-\tau_{1} / q\right) q^{\prime}} \phi_{\gamma}^{1-q^{\prime}} d x d t\right)^{1 / q^{\prime}}\right.} \\
& \left.+\left(\int_{D}\left|\Delta \phi_{\gamma}\right|^{q^{\prime}}|x|^{\tau_{1}\left(q^{\prime}-1\right)} \phi_{\gamma}^{1-q^{\prime}} d x d t\right)^{1 / q^{\prime}}\right] \Im_{1}^{1 / q}-\int_{\mathbb{R}^{N}} v_{0}|x|^{-\tau_{2}} \phi_{\gamma}(x, 0) d x .
\end{aligned}
$$

Using the definition of $\phi_{\gamma}$ and applying the following change of variables:

$$
t=R^{\gamma} s, \quad x=R \xi
$$

to the integrals in (2.8) and (2.9), we get

$$
\begin{gathered}
\int_{D}\left|\left(\phi_{\gamma}\right)_{t}\right|^{p^{\prime}}|x|^{-\left(\tau_{1}-\tau_{2} / p\right) p^{\prime}} \phi_{\gamma}^{1-p^{\prime}} d x d t \leq C R^{-\gamma p^{\prime}-\left(\tau_{1}-\tau_{2} / p\right) p^{\prime}+\gamma+N}, \\
\int_{D}\left|\Delta \phi_{\gamma}\right|^{p^{\prime}}|x|^{\tau_{2}\left(p^{\prime}-1\right)} \phi_{\gamma}^{1-p^{\prime}} d x d t \leq C R^{-2 p^{\prime}+\tau_{2}\left(p^{\prime}-1\right)+\gamma+N} .
\end{gathered}
$$

Analogously from (2.9) we get

$$
\begin{gathered}
\int_{D}\left|\left(\phi_{\gamma}\right)_{t}\right|^{q^{\prime}}|x|^{-\left(\tau_{2}-\tau_{1} / q\right) q^{\prime}} \phi_{\gamma}^{1-q^{\prime}} d x d t \leq C R^{-\gamma q^{\prime}-\left(\tau_{2}-\tau_{1} / q\right) q^{\prime}+\gamma+N}, \\
\int_{D}\left|\Delta \phi_{\gamma}\right|^{q^{\prime}}|x|^{\tau_{1}\left(q^{\prime}-1\right)} \phi_{\gamma}^{1-q^{\prime}} d x d t \leq C R^{-2 q^{\prime}+\tau_{1}\left(q^{\prime}-1\right)+\gamma+N} .
\end{gathered}
$$

We conclude the proof by substituting (2.11) and (2.12) into (2.8) and (2.9). 
For the sake of brevity, we introduce the following notations: for any $u_{0}, v_{0}$ such that $v_{0}|x|^{-\tau_{2}}, u_{0}|x|^{-\tau_{1}} \in L_{\text {loc }}^{1}\left(\mathbb{R}^{N}\right)$ and $\phi_{\gamma}$ given by (2.4) we set

$$
\begin{aligned}
& i_{1}(R)=\int_{\mathbb{R}^{N}} v_{0}(x)|x|^{-\tau_{2}} \phi_{\gamma}(x, 0) d x, \\
& i_{2}(R)=\int_{\mathbb{R}^{N}} u_{0}(x)|x|^{-\tau_{1}} \phi_{\gamma}(x, 0) d x .
\end{aligned}
$$

From Proposition 2.2 the following result follows.

Corollary 2.3. Assume that $v_{0}|x|^{-\tau_{2}}, u_{0}|x|^{-\tau_{1}} \in L_{\text {loc }}^{1}\left(\mathbb{R}^{N}\right)$. Let $(u, v)$ be a weak solution of (2.1). Then, for any $\phi_{\gamma} \in C_{0}^{\infty}\left(\mathbb{R}^{N} \times[0, \infty)\right)$ given by (2.4) the following estimates hold:

$$
\begin{aligned}
& \Phi_{1} \leq C\left(R^{\alpha_{1}}+R^{\alpha_{2}}\right)\left(\left(R^{\alpha_{3}}+R^{\alpha_{4}}\right) \Phi_{1}^{1 / q}-i_{1}(R)\right)^{1 / p}-i_{2}(R), \\
& \Phi_{2} \leq C\left(R^{\alpha_{3}}+R^{\alpha_{4}}\right)\left(\left(R^{\alpha_{1}}+R^{\alpha_{2}}\right) \Im_{2}^{1 / p}-i_{2}(R)\right)^{1 / q}-i_{1}(R) .
\end{aligned}
$$

Proof. The inequalities (2.14) and (2.15) follow from (2.7) by substitution.

Now we are in a position to state the main result of this section.

Theorem 2.4. Let $p, q>1$ and $\tau_{1} \leq \tau_{2}<2$. Assume that $u_{0}{ }^{-}|x|^{-\tau_{1}}, v_{0}{ }^{-}|x|^{-\tau_{2}} \in$ $L^{1}\left(\mathbb{R}^{N}\right)$ and that

$$
\liminf _{R \rightarrow \infty} \int_{B_{R}} u_{0}|x|^{-\tau_{1}} d x>0, \quad \liminf _{R \rightarrow \infty} \int_{B_{R}} v_{0}|x|^{-\tau_{2}} d x>0 .
$$

If

$$
\begin{aligned}
\min \{ & \left(N-\tau_{1}\right)(p q-1)-\left(2-\tau_{1}\right)-q\left(2-\tau_{2}\right), \\
& \left(N-\tau_{2}\right)(p q-1)-\left(2-\tau_{2}\right)-p\left(2-\tau_{2}\right), \\
& \left.\left(N-\tau_{1}\right)(p q-1)-\left(2-\tau_{2}\right)-p\left(2-\tau_{1}\right)\right\} \leq 0,
\end{aligned}
$$

then there exists no weak solution of (2.1).

Proof. Let $(u, v)$ be a weak solution of (2.1) and suppose that $i_{1}(R) \geq 0$ and $i_{2}(R) \geq 0$ for any $R$ large enough. Slight modifications yield the proof in the general case. From Corollary 2.3 it follows that

$$
\begin{aligned}
& \mathscr{g}_{1}^{1-(1 / p q)} \leq C\left(R^{\alpha_{1}}+R^{\alpha_{2}}\right)\left(R^{\alpha_{3}}+R^{\alpha_{4}}\right)^{1 / p}, \\
& \mathscr{g}_{2}^{1-(1 / p q)} \leq C\left(R^{\alpha_{3}}+R^{\alpha_{4}}\right)\left(R^{\alpha_{1}}+R^{\alpha_{2}}\right)^{1 / q} .
\end{aligned}
$$

We deduce that if there exists $\gamma>0$ such that one of the following conditions holds:

$$
\begin{aligned}
& \max \left\{\alpha_{1} p+\alpha_{3}, \alpha_{1} p+\alpha_{4}, \alpha_{2} p+\alpha_{3}, \alpha_{2} p+\alpha_{4}\right\}<0, \\
& \max \left\{\alpha_{1}+\alpha_{3} q, \alpha_{1}+\alpha_{4} q, \alpha_{2}+\alpha_{3} q, \alpha_{2}+\alpha_{4} q\right\}<0,
\end{aligned}
$$


then taking the limit as $R \rightarrow \infty$ in (2.18), or respectively in (2.19), we obtain that

$$
\int_{A_{\gamma, R}}|v|^{q}|x|^{-\tau_{1}} d x d t \longrightarrow 0, \quad \text { or respectively } \int_{A_{\gamma, R}}|u|^{p}|x|^{-\tau_{2}} d x d t \longrightarrow 0,
$$

where $A_{\gamma, R}=\left\{(x, t) \in D: t R^{-\gamma}+|x|^{2} R^{-2} \leq 1\right\}$. This implies that $u \equiv 0$ and $v \equiv 0$, against our assumption.

Now, define

$$
\begin{aligned}
& f_{1}(\gamma) \equiv \alpha_{1} p+\alpha_{3}, \quad f_{2}(\gamma) \equiv \alpha_{1} p+\alpha_{4}, \\
& f_{3}(\gamma) \equiv \alpha_{2} p+\alpha_{3}, \quad f_{4}(\gamma) \equiv \alpha_{2} p+\alpha_{4}, \\
& h_{1}(\gamma) \equiv \alpha_{1}+\alpha_{3} q, \quad h_{2}(\gamma) \equiv \alpha_{1}+\alpha_{4} q \text {, } \\
& h_{3}(\gamma) \equiv \alpha_{2}+\alpha_{3} q, \quad h_{4}(\gamma) \equiv \alpha_{2}+\alpha_{4} q .
\end{aligned}
$$

Using (2.6), it is easy to check that the lines $\delta=f_{i}(\gamma)$, and respectively $\delta=h_{i}(\gamma)$, mutually intersect at $\gamma=2-\tau_{1}$ and $\gamma=2-\tau_{2}$ as follows:

$$
\begin{aligned}
& f_{1}\left(2-\tau_{1}\right)=f_{3}\left(2-\tau_{1}\right)=\frac{\left(N-\tau_{1}\right)(p q-1)-\left(2-\tau_{1}\right)-q\left(2-\tau_{1}\right)}{q}, \\
& f_{2}\left(2-\tau_{1}\right)=f_{4}\left(2-\tau_{1}\right)=\frac{\left(N-\tau_{1}\right)(p q-1)-\left(2-\tau_{1}\right)-q\left(2-\tau_{2}\right)}{q}, \\
& f_{1}\left(2-\tau_{2}\right)=f_{2}\left(2-\tau_{2}\right)=\frac{\left(N-\tau_{1}\right)(p q-1)-\left(2-\tau_{2}\right)-q\left(2-\tau_{2}\right)}{q}, \\
& f_{3}\left(2-\tau_{2}\right)=f_{4}\left(2-\tau_{2}\right)=\frac{\left(N-\tau_{2}\right)(p q-1)-\left(2-\tau_{1}\right)-q\left(2-\tau_{2}\right)}{q}, \\
& h_{1}\left(2-\tau_{1}\right)=h_{3}\left(2-\tau_{1}\right)=\frac{\left(N-\tau_{2}\right)(p q-1)-\left(2-\tau_{1}\right)-p\left(2-\tau_{1}\right)}{p}, \\
& h_{2}\left(2-\tau_{1}\right)=h_{4}\left(2-\tau_{1}\right)=\frac{\left(N-\tau_{1}\right)(p q-1)-\left(2-\tau_{2}\right)-p\left(2-\tau_{1}\right)}{p}, \\
& h_{1}\left(2-\tau_{2}\right)=h_{2}\left(2-\tau_{2}\right)=\frac{\left(N-\tau_{2}\right)(p q-1)-\left(2-\tau_{2}\right)-p\left(2-\tau_{2}\right)}{p}, \\
& h_{3}\left(2-\tau_{2}\right)=h_{4}\left(2-\tau_{2}\right)=\frac{\left(N-\tau_{2}\right)(p q-1)-\left(2-\tau_{2}\right)-p\left(2-\tau_{2}\right)}{p} .
\end{aligned}
$$

Figure 2.1 shows the graphs of the lines $\delta=f_{i}(\gamma)$ for the following choice of the parameters: $p=2, q=3, \tau_{1}=0$, and $\tau_{2}=1$. Note that in this case $2-\tau_{1}=2$ and $2-\tau_{2}=1$.

The best condition on the parameters $p, q, \tau_{1}, \tau_{2}$ in order that (2.20a), or (2.20b) holds for some $\gamma>0$, (actually, $\gamma=2-\tau_{1}$ or $\gamma=2-\tau_{2}$ ), is

$$
\begin{aligned}
& \min \left\{\max \left\{f_{i}\left(2-\tau_{1}\right), i=1,4\right\}, \max \left\{f_{i}\left(2-\tau_{2}\right), i=1,4\right\},\right. \\
&\left.\max \left\{h_{i}\left(2-\tau_{1}\right), i=1,4\right\}, \max \left\{h_{i}\left(2-\tau_{2}\right), i=1,4\right\}\right\}<0 .
\end{aligned}
$$




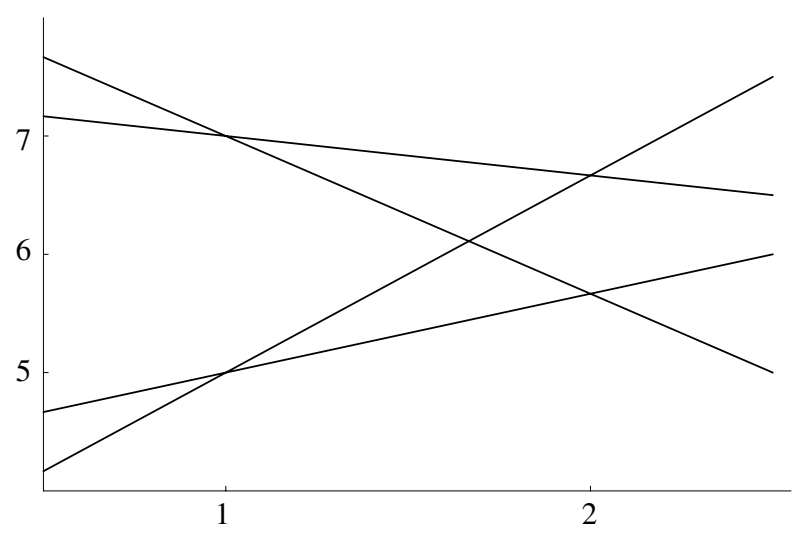

Figure 2.1

From the explicit expressions of $f_{i}\left(2-\tau_{j}\right)$ and $h_{i}\left(2-\tau_{j}\right)$ for $i=1,4$ and $j=1,2$ which can be obtained using (2.6) we can deduce that, since $\tau_{1} \leq \tau_{2}$,

$$
\begin{array}{ll}
f_{3}\left(2-\tau_{2}\right) \leq f_{2}\left(2-\tau_{2}\right), & f_{1}\left(2-\tau_{1}\right) \leq f_{2}\left(2-\tau_{1}\right), \\
h_{3}\left(2-\tau_{2}\right) \leq h_{2}\left(2-\tau_{2}\right), & h_{3}\left(2-\tau_{1}\right) \leq h_{2}\left(2-\tau_{1}\right) .
\end{array}
$$

Moreover, we can check that $f_{2}$ is decreasing and hence (2.24) is equivalent to

$$
\min \left\{f_{2}\left(2-\tau_{1}\right), h_{2}\left(2-\tau_{2}\right), h_{2}\left(2-\tau_{1}\right)\right\}<0,
$$

that is,

$$
\begin{aligned}
\min \{ & \left(N-\tau_{1}\right)(p q-1)-\left(2-\tau_{1}\right)-q\left(2-\tau_{2}\right), \\
& \left(N-\tau_{2}\right)(p q-1)-\left(2-\tau_{2}\right)-p\left(2-\tau_{2}\right), \\
& \left.\left(N-\tau_{1}\right)(p q-1)-\left(2-\tau_{2}\right)-p\left(2-\tau_{1}\right)\right\}<0 .
\end{aligned}
$$

This concludes the proof when (2.17) holds with the strict inequality.

Now, suppose that

$$
\begin{aligned}
\min \{ & \left(N-\tau_{1}\right)(p q-1)-\left(2-\tau_{1}\right)-q\left(2-\tau_{2}\right), \\
& \left(N-\tau_{2}\right)(p q-1)-\left(2-\tau_{2}\right)-p\left(2-\tau_{2}\right), \\
& \left.\left(N-\tau_{1}\right)(p q-1)-\left(2-\tau_{2}\right)-p\left(2-\tau_{1}\right)\right\} \\
= & \left(N-\tau_{1}\right)(p q-1)-\left(2-\tau_{1}\right)-q\left(2-\tau_{2}\right)=0 .
\end{aligned}
$$

The other cases can be handled similarly.

Since $\left(N-\tau_{1}\right)(p q-1)-\left(2-\tau_{1}\right)-q\left(2-\tau_{2}\right)=\max \left\{f_{i}\left(2-\tau_{1}\right), i=1,4\right\}=0$, it follows that for any $i=1,4$ we have $f_{i}\left(2-\tau_{1}\right) \leq 0$. Set $\gamma=2-\tau_{1}$. From (2.14), we get that

$$
\int_{D}|v|^{q}|x|^{-\tau_{1}} d x d t<\infty .
$$


272 Existence and nonexistence of global solutions

From the definition of weak solution and the fact that $\phi_{0}(s)=1$ for any $s \in[0,1]$, we know that

$$
\begin{aligned}
& \int_{D}|v|^{q}|x|^{-\tau_{1}} \phi_{2-\tau_{1}} d x d t \\
& \quad \leq-\int_{D_{R}} u\left(\Delta \phi_{2-\tau_{1}}+|x|^{-\tau_{1}}\left(\phi_{2-\tau_{1}}\right)_{t}\right) d x d t-\int_{\mathbb{R}^{N}} u_{0}|x|^{-\tau_{1}} \phi_{2-\tau_{1}} d x \\
& \int_{D}|u|^{p}|x|^{-\tau_{2}} \phi_{2-\tau_{1}} d x d t \\
& \quad \leq-\int_{D_{R}} v\left(\Delta \phi_{2-\tau_{1}}+|x|^{-\tau_{2}}\left(\phi_{2-\tau_{1}}\right)_{t}\right) d x d t-\int_{\mathbb{R}^{N}} v_{0}|x|^{-\tau_{2}} \phi_{2-\tau_{1}} d x
\end{aligned}
$$

where

$$
D_{R}=\left\{(x, t) \in D: 1<t R^{\tau_{1}-2}+|x|^{2} R^{-2}<2\right\} .
$$

Applying Hölder inequality and proceeding as in the proof of Proposition 2.2 and Corollary 2.3, we find that

$$
\int_{A_{\gamma, R}}|v|^{q}|x|^{-\tau_{1}} d x d t \leq C\left(\int_{D_{R}}|v|^{q}|x|^{-\tau_{1}} d x d t\right)^{1 /(p q)},
$$

where we have also used that $\phi_{2-\tau_{1}}(\cdot, \cdot) \leq 1$. From (2.29) we know that

$$
\int_{D_{R}}|v|^{q}|x|^{-\tau_{1}} d x d t \longrightarrow 0, \quad R \longrightarrow \infty
$$

hence taking the limit for $R \rightarrow \infty$ in (2.32) we get a contradiction. This concludes the proof.

Remark 2.5. If $\tau_{1}=\tau_{2}, p=q$, and $u_{0} \equiv v_{0}$, Theorem 2.4 recovers the result for the single inequality proved in [5]. For the sake of completeness we state the corresponding result.

Corollary 2.6. Let $p>1$ and $\tau<2$. Assume that $u_{0}{ }^{-}|x|^{-\tau} \in L^{1}\left(\mathbb{R}^{N}\right)$, and that

$$
\liminf _{R \rightarrow \infty} \int_{B_{R}} u_{0}|x|^{-\tau} d x>0 \text {. }
$$

If

$$
p \leq 1+\frac{2-\tau}{N-\tau},
$$

then there exists no weak solution of the following problem:

$$
\begin{gathered}
u_{t}-|x|^{\tau} \Delta u \geq|u|^{p}, \quad(x, t) \in D, \\
u(x, 0)=u_{0}(x), \quad x \in \mathbb{R}^{N} .
\end{gathered}
$$


Remark 2.7. In the special case $\tau_{1}=\tau_{2}=0$ the system (2.1) reduces to

$$
\begin{array}{cc}
u_{t}-\Delta u \geq|v|^{q}, & (x, t) \in D, \\
v_{t}-\Delta v \geq|u|^{p}, & (x, t) \in D, \\
u(x, 0)=u_{0}(x), & x \in \mathbb{R}^{N}, \\
v(x, 0)=v_{0}(x), & x \in \mathbb{R}^{N},
\end{array}
$$

and the nonexistence condition given by Theorem 2.4 coincides with the condition found by Escobedo and Herrero in [2], for the system of equations with positive initial data, that is,

$$
\max \left\{\frac{p+1}{p q-1}, \frac{q+1}{p q-1}\right\} \geq \frac{N}{2} .
$$

Now, consider the case when $\tau_{2}=2$ :

$$
\begin{gathered}
u_{t}-|x|^{\tau_{1}} \Delta u \geq|v|^{q}, \quad(x, t) \in D, \\
v_{t}-|x|^{2} \Delta v \geq|u|^{p}, \quad(x, t) \in D, \\
u(x, 0)=u_{0}(x), \quad x \in \mathbb{R}^{N}, \\
v(x, 0)=v_{0}(x), \quad x \in \mathbb{R}^{N} .
\end{gathered}
$$

Arguing as in the proof of Theorem 2.4 we can prove the following theorem.

Theorem 2.8. Let $p, q>1$ and $\tau_{1}<2$. Assume that $u_{0}{ }^{-}|x|^{-\tau_{1}}, v_{0}{ }^{-}|x|^{-2} \in L^{1}\left(\mathbb{R}^{N}\right)$ and that

$$
\liminf _{R \rightarrow \infty} \int_{B_{R}} u_{0}|x|^{-\tau_{1}} d x>0, \quad \liminf _{R \rightarrow \infty} \int_{B_{R}} v_{0}|x|^{-2} d x>0
$$

If

$$
\left(N-\tau_{1}\right)(p q-1)-\left(2-\tau_{2}\right)-p\left(2-\tau_{1}\right) \leq 0,
$$

then there exists no weak solution of (2.39).

Proof. The proof is similar to the proof of Theorem 2.4. In this case we are forced to choose $\gamma=2-\tau_{1}$. Hence, the best condition which guarantees that (2.20a) or (2.20b) holds is

$$
\min \left\{\max \left\{f_{i}\left(2-\tau_{1}\right)\right\}, \max \left\{h_{i}\left(2-\tau_{1}\right)\right\}\right\}<0,
$$

which taking into account of the explicit values of the $f_{i}\left(2-\tau_{1}\right)$ and $f_{i}\left(2-\tau_{1}\right)$ is equivalent to

$$
\left(N-\tau_{1}\right)(p q-1)-p\left(2-\tau_{1}\right)<0 .
$$

The case when $\left(N-\tau_{1}\right)(p q-1)-p\left(2-\tau_{1}\right)=0$ can be dealt with as in Theorem 2.4. 


\section{Systems of parabolic differential inequalities containing critical degeneracies}

3.1. Nonexistence of global solutions. Set $D^{*}=\mathbb{R}^{N} \backslash\{0\} \times(0, \infty)$. Consider the following initial value problem

$$
\begin{array}{cc}
u_{t}-|x|^{2} \Delta u \geq|v|^{q}, & (x, t) \in D^{*}, \\
v_{t}-|x|^{2} \Delta v \geq|u|^{p}, & (x, t) \in D^{*}, \\
u(x, 0)=u_{0}(x), & x \in \mathbb{R}^{N} \backslash\{0\}, \\
v(x, 0)=v_{0}(x), & x \in \mathbb{R}^{N} \backslash\{0\},
\end{array}
$$

where $p, q>1$ and $v_{0}, u_{0} \in L_{\text {loc }}^{1}\left(\mathbb{R}^{N} \backslash\{0\}\right)$.

Here, we extend to the case of systems the definition of weak solution introduced in [5] for critical degenerate problems.

Definition 3.1. We say that $(u, v)$ is a weak solution of (3.1) if the following assumptions are satisfied:

(i) $u, v: D^{*} \rightarrow \mathbb{R}$

(ii) $|v|^{q},|u|^{p} \in L_{\text {loc }}^{1}\left(D^{*}\right)$,

and for any nonnegative $\phi \in C_{0}^{\infty}\left(\mathbb{R}^{N} \backslash\{0\} \times[0,+\infty)\right)$ the following inequalities hold:

$$
\begin{aligned}
& \int_{D^{*}}|v|^{q}|x|^{-N} \phi d x d t \\
& \quad \leq-\int_{D^{*}} u\left(\Delta\left(|x|^{2-N} \phi\right)+|x|^{-N} \phi_{t}\right) d x d t-\int_{\mathbb{R}^{N}} u_{0}|x|^{-N} \phi(x, 0) d x, \\
& \int_{D^{*}}|u|^{p}|x|^{-N} \phi d x d t \\
& \quad \leq-\int_{D^{*}} v\left(\Delta\left(|x|^{2-N} \phi\right)+|x|^{-N} \phi_{t}\right) d x d t-\int_{\mathbb{R}^{N}} v_{0}|x|^{-N} \phi(x, 0) d x .
\end{aligned}
$$

It is understood that in the proofs of the theorems of this section we choose the cut-off function $\phi$ as follows: let $\psi_{0}, \psi_{1} \in C_{0}^{\infty}(\mathbb{R})$ be such that $0 \leq \psi_{i}(s) \leq 1$, for any $s \in \mathbb{R}, i=0,1$ and

$$
\psi_{0}(s)=\left\{\begin{array}{ll}
1, & 0 \leq s \leq 1, \\
0, & s \geq 2,
\end{array} \quad \psi_{1}(s)= \begin{cases}1, & |s| \leq 1, \\
0, & |s| \geq 2 .\end{cases}\right.
$$

Then for any $R>0$, we take

$$
\phi(x, t)=\psi_{0}\left(\frac{t}{R^{2}}\right) \psi_{1}\left(\frac{\log |x|+(N-2) t}{R}\right) .
$$

For the sake of brevity, we introduce the following notations: for any $u, v: D^{*} \rightarrow \mathbb{R}$ such that $|v|^{q}$ and $|u|^{p} \in L_{\text {loc }}^{1}\left(D^{*}\right)$ and $\phi \in C_{0}^{\infty}\left(\mathbb{R}^{N} \backslash\{0\} \times[0,+\infty)\right)$, we set

$$
\mathscr{F}_{1}=\int_{D^{*}}|v|^{q}|x|^{-N} \phi d x d t, \quad \mathscr{F}_{2}=\int_{D^{*}}|u|^{p}|x|^{-N} \phi d x d t .
$$


Proposition 3.2. Assume that $v_{0}, u_{0} \in L_{\text {loc }}^{1}\left(\mathbb{R}^{N} \backslash\{0\}\right)$ and that $(u, v)$ is a weak solution of (3.1). Then, for any $\phi \in C_{0}^{\infty}\left(\mathbb{R}^{N} \backslash\{0\} \times[0,+\infty)\right)$ given by (3.4) the following estimates hold:

$$
\begin{aligned}
& \mathscr{F}_{1} \leq C R^{\left(3-2 p^{\prime}\right) / p^{\prime}} \mathscr{g}_{2}^{1 / p}-\int_{\mathbb{R}^{N}} u_{0}|x|^{-N} \phi(x, 0) d x, \\
& \mathscr{F}_{2} \leq C R^{\left(3-2 q^{\prime}\right) / q^{\prime}} \mathscr{g}_{1}^{1 / q}-\int_{\mathbb{R}^{N}} v_{0}|x|^{-N} \phi(x, 0) d x .
\end{aligned}
$$

Proof. Suppose that $N>2$ and let $(u, v)$ be a weak solution of (3.1). We proceed as in the proof of Proposition 2.2. First we apply Hölder inequality to the right-hand sides of (3.2) with $\phi$ given by (3.4) and obtain

$$
\begin{aligned}
\mathscr{E}_{1} \leq & \mathscr{g}_{2}^{1 / p}\left(\int_{D^{*}} \frac{\left.\left.\left|\Delta\left(|x|^{2-N} \phi\right)+\phi_{t}\right| x\right|^{-N}\right|^{p^{\prime}}}{\phi^{p^{\prime}-1}}|x|^{N\left(p^{\prime}-1\right)} d x d t\right)^{1 / p^{\prime}} \\
& -\int_{\mathbb{R}^{N}} u_{0}|x|^{-N} \phi(x, 0) d x, \\
\mathscr{I}_{2} \leq & \mathscr{g}_{1}^{1 / q}\left(\int_{D^{*}} \frac{\left.\left.\left|\Delta\left(|x|^{2-N} \phi\right)+\phi_{t}\right| x\right|^{-N}\right|^{q^{\prime}}}{\phi^{q^{\prime}-1}}|x|^{N\left(q^{\prime}-1\right)} d x d t\right)^{1 / q^{\prime}} \\
& -\int_{\mathbb{R}^{N}} v_{0}|x|^{-N} \phi(x, 0) d x .
\end{aligned}
$$

In order to estimate the term

$$
\int_{D^{*}} \frac{\left.\left.\left|\Delta\left(|x|^{2-N} \phi\right)+\phi_{t}\right| x\right|^{-N}\right|^{p^{\prime}}}{\phi^{p^{\prime}-1}} d x d t
$$

we first apply the change of variables

$$
\sigma=\log (|x|), \quad|x|>0
$$

and obtain

$$
\int_{D^{*}} \frac{\left.\left.\left|\Delta\left(|x|^{2-N} \phi\right)+\phi_{t}\right| x\right|^{-N}\right|^{p^{\prime}}}{\phi^{p^{\prime}-1}} d x d t \leq C \int_{0}^{\infty} \int_{-\infty}^{\infty} \frac{\left|\phi_{\sigma \sigma}+(2-N) \phi_{\sigma}+\phi_{t}\right|^{p^{\prime}}}{\phi^{p^{\prime}-1}} d \sigma d t .
$$

Then, we introduce the change of variables,

$$
t=R^{2} \tau, \quad \sigma=R \xi,
$$

and we get

$$
\begin{aligned}
\int_{0}^{\infty} \int_{-\infty}^{\infty} & \frac{\left|\phi_{\sigma \sigma}+(2-N) \phi_{\sigma}+\phi_{t}\right|^{p^{\prime}}}{\phi^{p^{\prime}-1}} d \sigma d t \\
& \leq C R^{3-2 p^{\prime}} \int_{0}^{\infty} \int_{-\infty}^{\infty} \Gamma(\xi, \tau)^{p^{\prime}} \phi(\xi, \tau)^{1-p^{\prime}} d \xi d \tau
\end{aligned}
$$


where

$$
\begin{gathered}
\Gamma(\xi, \tau)=\left|\psi_{0}(\tau) \psi_{1}^{\prime \prime}(\xi+(N-2) R \tau)+\psi_{0}^{\prime}(\tau) \psi_{1}(\xi+(N-2) R \tau)\right|, \\
\phi(\xi, \tau)=\psi_{0}(\tau) \psi_{1}(\xi+(N-2) R \tau) .
\end{gathered}
$$

Analogously, we obtain

$$
\begin{aligned}
& \int_{D} \frac{\left.\left.\left|\Delta\left(|x|^{2-N} \phi\right)+\phi_{t}\right| x\right|^{-N}\right|^{q^{\prime}}}{\phi^{q^{\prime}-1}} d x d t \\
& \quad \leq C R^{3-2 q^{\prime}} \int_{0}^{\infty} \int_{-\infty}^{\infty} \Gamma(\xi, \tau)^{q^{\prime}} \phi(\xi, \tau)^{1-q^{\prime}} d \xi d \tau .
\end{aligned}
$$

Now, we observe that since the functions $\psi_{i}$ for $i=0,1$ have compact support, then

$$
\begin{aligned}
& \int_{0}^{\infty} \int_{-\infty}^{\infty} \Gamma(\xi, \tau)^{p^{\prime}} \phi(\xi, \tau)^{1-p^{\prime}} d \xi d \tau<\infty, \\
& \int_{0}^{\infty} \int_{-\infty}^{\infty} \Gamma(\xi, \tau)^{q^{\prime}} \phi(\xi, \tau)^{1-q^{\prime}} d \xi d \tau<\infty .
\end{aligned}
$$

From (3.12) and (3.14) the statement follows.

Set

$$
j_{1}(R)=\int_{\mathbb{R}^{N}} u_{0}|x|^{-N} \phi(x, 0) d x \quad j_{2}(R)=\int_{\mathbb{R}^{N}} v_{0}|x|^{-N} \phi(x, 0) d x,
$$

where $\phi$ is given by (3.4). Notice that $j_{i}(\cdot),(i=1,2)$, depend on $R$ through $\phi$.

Corollary 3.3. Let $(u, v)$ be a weak solution of (3.1). Assume that $v_{0}, u_{0} \in L_{\text {loc }}^{1}\left(\mathbb{R}^{N} \backslash\right.$ $\{0\})$. Then, for any $\phi \in C_{0}^{\infty}\left(\mathbb{R}^{N} \backslash\{0\} \times[0,+\infty)\right)$ given by (3.4) the following estimates hold:

$$
\begin{aligned}
& \mathscr{g}_{1} \leq C R^{\left(3-2 p^{\prime}\right) / p^{\prime}}\left(C R^{\left(3-2 q^{\prime}\right) /\left(q^{\prime} p\right)} \mathscr{g}_{1}^{1 / q}-j_{1}(R)\right)^{1 / p}-j_{2}(R), \\
& \mathscr{g}_{2} \leq C R^{\left(3-2 q^{\prime}\right) / q^{\prime}}\left(C R^{\left(3-2 p^{\prime}\right) /\left(p^{\prime} p\right)} \mathscr{g}_{2}^{1 / p}-j_{2}(R)\right)^{1 / q}-j_{1}(R) .
\end{aligned}
$$

Proof. The inequalities (3.17) follow from (3.6) by substitution.

Theorem 3.4. Let $p, q>1$. Assume that $u_{0}{ }^{-}, v_{0}{ }^{-} \in L_{\mathrm{loc}}^{1}\left(\mathbb{R}^{N} \backslash\{0\}\right)$ and that

$$
\liminf _{R \rightarrow \infty} \int_{B_{R}} u_{0}|x|^{-N} d x>0, \quad \liminf _{R \rightarrow \infty} \int_{B_{R}} v_{0}|x|^{-N} d x>0 .
$$

If one of the following conditions holds:

$$
\begin{aligned}
& q(p-2) \leq 3, \\
& p(q-2) \leq 3,
\end{aligned}
$$

then there exists no weak solution of problem (3.1). 
Proof. Let $(u, v)$ be a weak solution of (3.1). Assume that $j_{1}(R) \geq 0$ and $j_{2}(R) \geq 0$ for any $R$ large enough. Slight modifications yield the proof in the general case. From Corollary 3.3, it follows that

$$
\begin{aligned}
& \left(\int_{D_{R}}|v|^{q}|x|^{-N} d x d t\right)^{1-1 /(p q)} \leq C R^{\left(3-2 p^{\prime}\right) /\left(p^{\prime}\right)+\left(3-2 q^{\prime}\right) /\left(q^{\prime} p\right)}, \\
& \left(\int_{D_{R}}|u|^{p}|x|^{-N} d x d t\right)^{1-1 /(p q)} \leq C R^{\left(3-2 q^{\prime}\right) /\left(q^{\prime}\right)+\left(3-2 p^{\prime}\right) /\left(p^{\prime} q\right)},
\end{aligned}
$$

where $D_{R}=\left\{(x, t) \in D^{*}:|\log | x|+(N-2) t| \leq R\right.$ and $\left.t \leq R^{2}\right\}$. If $q(p-2)<3$, then

$$
\frac{3-2 p^{\prime}}{p^{\prime}}+\frac{3-2 q^{\prime}}{q^{\prime} p}<0
$$

From (3.21) we conclude that

$$
\int_{B_{R}}|v|^{q}|x|^{-N} d x d t \longrightarrow 0, \quad \text { as } R \longrightarrow \infty,
$$

against our assumption that $v \neq 0$. If $q(p-2)=3$, we proceed as in the proof of Theorem 2.4. We argue similarly if (3.20) holds. This completes the proof.

Remark 3.5. When $p=q>1$, the condition (3.19) is equivalent to $p \leq 3$. Hence, Theorem 3.4 contains the result for the single inequality proved by Mitidieri and Pohozaev in [5], see also Giacomoni [3] for the case of the equation with positive initial data.

3.2. Existence of global solutions. In this section, we deal with the problem of the existence of global solutions of system (3.1), when we assume that $p, q>1$ do not satisfy (3.19) or (3.20) and the initial data $u_{0}$ and $v_{0}$ are nonnegative, radially symmetric and small. The result we are going to prove (see Theorem 3.6) shows that the curve in the $(p, q)$-plane defined by

$$
\min \{q(p-2), p(q-2)\}=3, \quad p, q>1,
$$

is the sharp critical curve for problem (3.1), that is,

(a) if $p, q>1$ and $\min \{q(p-2), p(q-2)\} \leq 3$, then there exists no weak solution defined on $D^{*}$;

(b) if $p, q>1$ and $\min \{q(p-2), p(q-2)\}>3$, then there exist global solutions defined on $\mathbb{R}^{N} \times(0, \infty)$ for sufficiently small initial data.

Part (a) has been established in Theorem 3.4. In order to prove part (b) we restrict our attention to radial solutions of (3.1). Hence, we assume that $u_{0}(x)=u_{0}(|x|)$ and $v_{0}(x)=v_{0}(|x|)$.

By introducing the change of variables $s=-\log (|x|)$ and setting $\tilde{u}(s, t)=$ $u(s, t) \exp (-(N-2) /(2) s)$ and $\tilde{v}(s, t)=v(s, t) \exp (-(N-2) /(2) s)$, it is easy to see 
that the radial solutions of system (2.39) satisfy

$$
\begin{array}{cc}
\tilde{u}_{t}-\tilde{u}_{s s}+\lambda_{N} \tilde{u}=e^{-((N-2) / 2)(q-1) s} \tilde{v}^{q}, & s \in \mathbb{R}, t>0, \\
\tilde{v}_{t}-\tilde{v}_{s s}+\lambda_{N} \tilde{v}=e^{-((N-2) / 2)(p-1) s} \tilde{u}^{p}, & s \in \mathbb{R}, t>0, \\
\tilde{u}(s, 0)=\tilde{u}_{0}(s), \quad \tilde{v}(s, 0)=\tilde{v}_{0}(s), \quad s \in \mathbb{R},
\end{array}
$$

where $\tilde{u}_{0}(s)=u_{0}(s) \exp (-((N-2) / 2) s), \quad \tilde{v}_{0}(s)=v_{0}(s) \exp (-((N-2) / 2) s)$ and $\lambda_{N}=((N-2) / 2)^{2}$.

\section{THEOREM 3.6. Assume that}

$$
\min \{q(p-2), p(q-2)\}>3 .
$$

Then the system (3.1) has global solutions for small initial data.

Proof. During the course we adapt the idea developed in [3] for the equation to the case of systems. In particular, in [3] it is proved that the heat kernel of the linear differential operator $-u_{s s}+\lambda_{N} u$ is given by

$$
H(s, t)=\frac{\exp \left(-\lambda_{N} t-s^{2} / 4 t\right)}{\sqrt{4 \pi t}}, \quad s \in \mathbb{R}, t>0 .
$$

Hence, the solutions of (3.26) satisfy the following integral system:

$$
\begin{aligned}
& \tilde{u}(s, t)=H(s, t) * \tilde{u}_{0}(s)+\int_{0}^{t} H(s, t-\tau) * \tilde{v}(s, \tau)^{q} e^{((N-2) / 2)(q-1) s} d \tau, \\
& \tilde{v}(s, t)=H(s, t) * \tilde{v}_{0}(s)+\int_{0}^{t} H(s, t-\tau) * \tilde{u}(s, \tau)^{p} e^{((N-2) / 2)(p-1) s} d \tau,
\end{aligned}
$$

where $*$ denotes the convolution operator in the space variable.

Now, we sketch the idea of the proof. Assume that (3.27) holds and that $q \geq p$. It follows that $q>3$. Assume that there exists $C>0$ such that

$$
\begin{array}{ll}
0 \leq \tilde{u}_{0}(s) \leq C H(s, \gamma), & s \in \mathbb{R}, \\
0 \leq \tilde{v}_{0}(s) \leq C H(s, \gamma), & s \in \mathbb{R} .
\end{array}
$$

Let

$$
\mathscr{X}=\left\{w(\cdot, t) \in L^{\infty}\left(\mathbb{R}^{N}\right): \exists K>0 \text { such that }|w(s, t)| \leq K H(s, t+\gamma)\right\} .
$$

$\mathscr{X}$ is a Banach space with respect to the norm

$$
|w|_{\mathscr{X}}=\sup _{t>0}\left|\frac{w(\cdot, t)}{H(\cdot, t+\gamma)}\right|_{\infty} .
$$

Define the operators

$$
\begin{aligned}
& \Phi_{1}(w)(s, t)=H(s, t) * \tilde{v}_{0}(s)+\int_{0}^{t} H(s, t-\tau) * w(s, \tau)^{p} e^{((N-2) / 2)(p-1) s} d \tau, \\
& \Phi_{2}(w)(s, t)=H(s, t) * \tilde{u}_{0}(s)+\int_{0}^{t} H(s, t-\tau) * w(s, \tau)^{q} e^{((N-2) / 2)(q-1) s} d \tau,
\end{aligned}
$$


where $w \in \mathscr{X}$. Since $H(\cdot, \cdot) \geq 0$ and $\tilde{u}_{0}, \tilde{v}_{0} \geq 0$, it follows that both $\Phi_{1}$ and $\Phi_{2}$ leave invariant the cone $P=\{w \in \mathscr{X}: w \geq 0\}$ of positive functions.

In what follows, we will prove that $\Phi_{2} \circ \Phi_{1}$ is a contraction on a small closed ball $B$ of $\mathscr{X}$. By the contraction mapping principle, it will follow that there exists $\bar{u} \in B \cap P$ such that

$$
\bar{u}=\Phi_{2} \circ \Phi_{1}(\bar{u}) .
$$

Defining $\bar{v}=\Phi_{1}(\bar{u})$, we obtain a solution $(\bar{u}, \bar{v})$ of the integral system (3.29). Note that if $\tilde{u}_{0}$ and $\tilde{v}_{0}$ are continuous, then $\bar{u}$ and $\bar{v}$ are smooth.

First, we prove that $\Phi_{2} \circ \Phi_{1}(\mathscr{X}) \subset \mathscr{X}$. To this aim we remark that from (3.30) it follows that

$$
H(s, t) * \tilde{u}_{0}(s) \leq C \int_{\mathbb{R}} H(s-x, t) H(x, \gamma) d x=C H(s, t+\gamma),
$$

and similarly from (3.31),

$$
H(s, t) * \tilde{v}_{0}(s) \leq C \int_{\mathbb{R}} H(s-x, t) H(x, \gamma) d x=C H(s, t+\gamma) .
$$

The following estimate will be useful:

$$
\begin{aligned}
& \int_{0}^{t} H(s, t-\tau) * H(s, \tau+\gamma)^{p} e^{((N-2) / 2)(p-1) s} d \tau \\
& \quad=\int_{0}^{t} H(s, t-\tau) * H(s, \tau+\gamma)\left(\frac{e^{-\lambda_{N}(\tau+\gamma)-\left(s^{2} / 4(\tau+\gamma)\right)+((N-2) / 2) s}}{\sqrt{4 \pi(\tau+\gamma)}}\right)^{p-1} d \tau \\
& \quad \leq H(s, t+\gamma) \int_{0}^{t}(4 \pi(\tau+\gamma))^{-(p-1) / 2} d \tau
\end{aligned}
$$

where we have used that $\sup \left\{e^{-\lambda_{N}(\tau+\gamma)-\left(s^{2} / 4(\tau+\gamma)\right)+((N-2) / 2) s}: s \in \mathbb{R}\right\}=1$.

Now, assume that

$$
0 \leq w(s, t) \leq C H(s, t+\gamma), \quad s \in \mathbb{R}, t>0 .
$$

From the definition of $\Phi_{1}$ and $\Phi_{2}$, we get that

$$
\begin{aligned}
& \Phi_{2}\left(\Phi_{1}(w)\right)(s, t) \\
& =H(s, t) * \tilde{u}_{0}(s)+\int_{0}^{t} H(s, t-\tau) *\left(\Phi_{1}(w)(s, \tau)\right)^{q} e^{((N-2) / 2)(q-1) s} d \tau \\
& \leq C H(s, t+\gamma)+2^{q-1} \int_{0}^{t} H(s, t-\tau) *\left(H(s, \tau) * v_{0}(s)\right)^{q} e^{((N-2) / 2)(q-1) s} d \tau \\
& \quad+2^{q-1} \int_{0}^{t} H(s, t-\tau) *\left(\int_{0}^{\tau} H(s, \tau-\eta) * w(s, \eta)^{p} e^{((N-2) / 2)(p-1) s} d \eta\right)^{q} \\
& \quad \times e^{((N-2) / 2)(q-1) s} d \tau .
\end{aligned}
$$


From (3.37) and (3.39), we have

$$
\begin{aligned}
\int_{0}^{t} H(s, t-\tau) *\left(H(s, \tau) * v_{0}(s)\right)^{q} e^{((N-2) / 2)(q-1) s} d \tau \\
\quad \leq C^{q} \int_{0}^{t} H(s, t-\tau) * H(s, \tau+\gamma)^{q} e^{((N-2) / 2)(q-1) s} d \tau \\
\quad \leq C^{q} H(s, t+\gamma) \int_{0}^{t}(4 \pi(\tau+\gamma))^{-(q-1) / 2} d \tau
\end{aligned}
$$

Since $q>3$ it follows that

$$
\int_{0}^{\infty}(4 \pi(\tau+\gamma))^{-(q-1) / 2} d \tau=K_{1}<\infty
$$

Using (3.42) in (3.41), we get that

$$
\int_{0}^{t} H(s, t-\tau) *\left(H(s, \tau) * v_{0}(s)\right)^{q} e^{((N-2) / 2)(q-1) s} d \tau \leq C^{q} K_{1} H(s, t+\gamma) .
$$

On the other hand, from (3.39) and (3.38), we obtain

$$
\begin{aligned}
& \int_{0}^{t} H(s, t-\tau) *\left(\int_{0}^{\tau} H(s, \tau-\eta) * w(s, \eta)^{p} e^{((N-2) / 2)(p-1) s} d \eta\right)^{q} e^{((N-2) / 2)(q-1) s} d \tau \\
& \leq C^{p q} \int_{0}^{t} H(s, t-\tau) * H(s, \tau+\gamma)^{q} e^{((N-2) / 2)(q-1) s} \\
& \times\left(\int_{0}^{\tau}(4 \pi(\eta+\gamma))^{-(p-1) / 2} d \eta\right)^{q} d \tau \\
& \leq C^{p q} H(s, t+\gamma) \cdot \int_{0}^{t}\left(\int_{0}^{\tau}(4 \pi(\eta+\gamma))^{-(q-1) / 2} d \eta\right) \\
& \times\left(\int_{0}^{\tau}(4 \pi(\eta+\gamma))^{-(p-1) / 2} d \eta\right)^{q} d \tau
\end{aligned}
$$

Since $q>3$ and $q(p-2)>3$, it follows that

$$
\int_{0}^{\infty}\left(\int_{0}^{\tau}(4 \pi(\eta+\gamma))^{-(q-1) / 2} d \eta\right)\left(\int_{0}^{\tau}(4 \pi(\eta+\gamma))^{-(p-1) / 2} d \eta\right)^{q} d \tau=K_{2}
$$

is finite. Therefore, from (3.43), (3.44), and (3.45) we conclude that

$$
\Phi_{2} \circ \Phi_{1}(w)(s, t) \leq C H(s, t+\gamma)+2^{q-1} C^{q} K_{1} H(s, t+\gamma)+2^{q-1} C^{p q} K_{2} H(s, t+\gamma),
$$

which shows that if $C>0$ is sufficiently small and $B_{C}$ denotes the ball of $\mathscr{X}$ of radius $C$, then $\Phi_{2} \circ \Phi_{1}\left(B_{C} \cap P\right) \subset B_{C} \cap P$. 
Now, we prove that $\Phi_{2} \circ \Phi_{1}$ is a contraction of $B_{C} \cap P$ for $C>0$ sufficiently small. Let $w_{1}$ and $w_{2} \in \mathscr{L}$ satisfy (3.39). Then, for any $t>0$, we deduce that

$$
\begin{aligned}
& \left|\Phi_{1}\left(w_{1}\right)(s, \tau)-\Phi_{1}\left(w_{2}\right)(s, \tau)\right| \\
& \leq \int_{0}^{\tau} H(s, \tau-\eta) *\left|w_{1}(s, \eta)^{p}-w_{2}(s, \eta)^{p}\right| e^{((N-2) / 2)(p-1) s} d \eta \\
& \leq p C^{p-1} \int_{0}^{\tau} H(s, \tau-\eta) * H(s, \eta+\gamma)^{p-1}\left|w_{1}(s, \eta)-w_{2}(s, \eta)\right| \\
& \quad \times e^{((N-2) / 2)(p-1) s} d \eta \\
& \leq p C^{p-1}\left\|w_{1}-w_{2}\right\|_{\mathscr{C}} H(s, \tau+\gamma) \int_{0}^{\tau}(4 \pi(\eta+\gamma))^{-(p-1) / 2} d \eta .
\end{aligned}
$$

Furthermore,

$$
\begin{aligned}
&\left|\Phi_{2} \circ \Phi_{1}\left(w_{1}\right)(s, t)-\Phi_{2} \circ \Phi_{1}\left(w_{2}\right)(s, t)\right| \\
& \leq \int_{0}^{t} H(s, t-\tau) *\left|\Phi_{1}\left(w_{1}\right)(s, \tau)^{q}-\Phi_{1}\left(w_{2}\right)(s, \tau)^{q}\right| e^{((N-2) / 2)(q-1) s} d \tau \\
& \leq q C^{p(q-1)} \int_{0}^{t} H(s, t-\tau) * H(s, \tau+\gamma)^{q-1}\left(\int_{0}^{\tau}(4 \pi(\eta+\gamma))^{-(p-1) / 2} d \eta\right)^{q-1} \\
& \times e^{((N-2) / 2)(q-1) s}\left|\Phi_{1}\left(w_{1}\right)(s, \tau)-\Phi_{1}\left(w_{2}\right)(s, \tau)\right| d \tau .
\end{aligned}
$$

Using (3.47) in (3.48) we obtain

$$
\begin{aligned}
\left|\Phi_{2} \circ \Phi_{1}\left(w_{1}\right)(s, t)-\Phi_{2} \circ \Phi_{1}\left(w_{2}\right)(s, t)\right| \\
\leq p q C^{p(q-1)+(p-1)\left\|w_{1}-w_{2}\right\|_{\mathscr{X}}} \\
\quad \times \int_{0}^{t} H(s, t-\tau) * H(s, \tau+\gamma)^{q}\left(\int_{0}^{\tau}(4 \pi(\eta+\gamma))^{-(p-1) / 2} d \eta\right)^{q} \\
\quad \times e^{((N-2) / 2)(q-1) s} d \tau \\
\leq p q C^{p(q-1)+(p-1)}\left\|w_{1}-w_{2}\right\|_{\mathscr{L}} H(s, t+\gamma) \\
\quad \times \int_{0}^{t}\left(\int_{0}^{\tau}(4 \pi(\eta+\gamma))^{-(p-1) / 2} d \eta\right)^{q}(4 \pi(\tau+\gamma))^{-(q-1) / 2} d \tau \\
\leq p q C^{p(q-1)+(p-1)} H(s, t+\gamma) K_{2}\left\|w_{1}-w_{2}\right\|_{\mathscr{L} .} .
\end{aligned}
$$

From (3.49), we get that

$$
\left\|\Phi_{2} \circ \Phi_{1}\left(w_{1}\right)-\Phi_{2} \circ \Phi_{1}\left(w_{2}\right)\right\|_{\mathscr{L}} \leq p q C^{p(q-1)+(p-1)} K_{2}\left\|w_{1}-w_{2}\right\|_{\mathscr{L}} .
$$

Hence, if $C>0$ is sufficiently small, $\Phi_{2} \circ \Phi_{1}$ is a contraction. This concludes the proof. 
282 Existence and nonexistence of global solutions

\section{Systems of singular parabolic equations}

Consider the following initial value problem:

$$
\begin{gathered}
u_{t}-\Delta u \geq t^{k_{1}}|x|^{-\sigma_{1}}|v|^{q}, \quad(x, t) \in D, \\
v_{t}-\Delta v \geq t^{k_{2}}|x|^{-\sigma_{2}}|u|^{p}, \quad(x, t) \in D, \\
u(x, 0)=u_{0}(x), \quad x \in \mathbb{R}^{N}, \\
v(x, 0)=v_{0}(x), \quad x \in \mathbb{R}^{N},
\end{gathered}
$$

where we assume that $k_{i}, \sigma_{i} \in \mathbb{R}$ for $i=1,2, p, q>1$ and $v_{0}, u_{0} \in L_{\text {loc }}^{1}\left(\mathbb{R}^{N}\right)$.

Definition 4.1. We say that $(u, v)$ is a weak solution of (4.1) if the following assumptions are satisfied:

(i) $u, v: D \rightarrow \mathbb{R}$

(ii) $|v|^{q} t^{k_{1}}|x|^{-\sigma_{1}},|u|^{p} t^{k_{2}}|x|^{-\sigma_{2}} \in L_{\mathrm{loc}}^{1}(D)$,

(iii) $u, v \in L_{\text {loc }}^{1}(D)$,

and for any nonnegative $\phi \in C_{0}^{\infty}\left(\mathbb{R}^{N} \times[0, \infty)\right)$ the following inequalities hold:

$$
\begin{aligned}
& \int_{D}|v|^{q} t^{k_{1}}|x|^{-\sigma_{1}} \phi d x d t \leq-\int_{D} u\left(\Delta \phi+\phi_{t}\right) d x d t-\int_{\mathbb{R}^{N}} u_{0} \phi(x, 0) d x, \\
& \int_{D}|u|^{p} t^{k_{2}}|x|^{-\sigma_{2}} \phi d x d t \leq-\int_{D} v\left(\Delta \phi+\phi_{t}\right) d x d t-\int_{\mathbb{R}^{N}} v_{0} \phi(x, 0) d x .
\end{aligned}
$$

Theorem 4.2. Let $p, q>1$. Assume that $u_{0}{ }^{-}, v_{0}{ }^{-} \in L_{\mathrm{loc}}^{1}\left(\mathbb{R}^{N}\right)$ and that

$$
\liminf _{R \rightarrow \infty} \int_{B_{R}} u_{0} d x>0, \quad \liminf _{R \rightarrow \infty} \int_{B_{R}} v_{0} d x>0 .
$$

If $k_{1}<q-1, k_{2}<p-1, \sigma_{2}+N(p-1)>0, \sigma_{1}+N(q-1)>0$ and

$$
\begin{aligned}
& \min \left\{N(p q-1)-2\left(q+1+k_{1}+k_{2} q\right)+\sigma_{1}+q \sigma_{2},\right. \\
&\left.N(p q-1)-2\left(p+1+k_{2}+k_{1} p\right)+\sigma_{2}+p \sigma_{1}\right\} \leq 0,
\end{aligned}
$$

then, there exists no weak solution of (4.1).

Proof. Let $(u, v)$ be a weak solution of (4.1). The proof is similar to that of Theorem 2.4, for this reason most of the details are omitted. Let $\phi=\phi_{\gamma}$ with $\phi_{\gamma}$ given by (2.4) and set

$$
\Phi_{1}=\int_{D}|v|^{q}|x|^{-\sigma_{1}} t^{k_{1}} \phi_{\gamma} d x d t, \quad \Phi_{2}=\int_{D}|u|^{p}|x|^{-\sigma_{2}} t^{k_{2}} \phi_{\gamma} d x d t .
$$

Further, assume that for any $R>0$ sufficiently large

$$
\int_{\mathbb{R}^{N}} u_{0} \phi(x, 0) d x \geq 0, \quad \int_{\mathbb{R}^{N}} v_{0} \phi(x, 0) d x \geq 0 .
$$


The general case can be treated by slight modifications.

Applying Hölder inequality to (4.2) we get

$$
\begin{aligned}
& \Phi_{1} \leq g_{2}^{1 / p}\left[\left(\int_{D}\left|\left(\phi_{\gamma}\right)_{t}\right|^{p^{\prime}}|x|^{\sigma_{2}\left(p^{\prime} / p\right)} t^{-k_{2}\left(p^{\prime} / p\right)} \phi_{\gamma}^{\left(p^{\prime} / p\right)} d x d t\right)^{1 / p^{\prime}}\right. \\
& \left.+\left(\int_{D}\left|\Delta \phi_{\gamma}\right|^{p^{\prime}}|x|^{\sigma_{2}\left(p^{\prime} / p\right)} t^{-k_{2}\left(p^{\prime} / p\right)} \phi_{\gamma}^{p^{\prime} / p} d x d t\right)^{1 / p^{\prime}}\right], \\
& \mathscr{I}_{2} \leq \mathscr{I}_{1}^{1 / q}\left[\left(\int_{D}\left|\left(\phi_{\gamma}\right)_{t}\right|^{q^{\prime}}|x|^{\sigma_{1}\left(q^{\prime} / q\right)} t^{-k_{1}\left(q^{\prime} / q\right)} \phi_{\gamma}^{q^{\prime} / q} d x d t\right)^{1 / q^{\prime}}\right. \\
& \left.+\left(\int_{D}\left|\Delta \phi_{\gamma}\right|^{q^{\prime}}|x|^{\sigma_{1}\left(q^{\prime} / q\right)} t^{-k_{1}\left(q^{\prime} / q\right)} \phi_{\gamma}^{q^{\prime} / q} d x d t\right)^{1 / q^{\prime}}\right] .
\end{aligned}
$$

Substituting (4.8) in (4.7) and proceeding as in Proposition 2.2 and Corollary 2.3 to estimate the integrals, we get

$$
\Phi_{1}^{1-1 /(p q)} \leq C\left(R^{\alpha_{1}}+R^{\alpha_{2}}\right)\left(R^{\alpha_{3}}+R^{\alpha_{4}}\right)^{1 / p},
$$

where

$$
\begin{array}{ll}
\alpha_{1}=\frac{N+\gamma}{p^{\prime}}-\frac{2+\sigma_{2}}{p}-\frac{k_{2}}{p}, & \alpha_{2}=\frac{N+\gamma}{p^{\prime}}-\frac{\gamma+\sigma_{2}}{p}-\frac{k_{2}}{p}, \\
\alpha_{3}=\frac{N+\gamma}{q^{\prime}}-\frac{2+\sigma_{1}}{q}-\frac{k_{1}}{q}, & \alpha_{4}=\frac{N+\gamma}{q^{\prime}}-\frac{\gamma+\sigma_{1}}{q}-\frac{k_{1}}{q} .
\end{array}
$$

It is easy to check that if $\gamma=2$, then

$$
\begin{aligned}
\alpha_{1} p+\alpha_{3} & =\alpha_{1} p+\alpha_{4}=\alpha_{2} p+\alpha_{3}=\alpha_{2} p+\alpha_{4} \\
& =\frac{N(p q-1)-2\left(q+1+k_{1}+k_{2} q\right)+\sigma_{1}+q \sigma_{2}}{q} .
\end{aligned}
$$

From (4.9) we conclude that if

$$
N(p q-1)-2\left(q+1+k_{1}+k_{2} q\right)+\sigma_{1}+q \sigma_{2}<0,
$$

then no weak solution of (4.1) exists. Analogously, substituting (4.7) in (4.8), we prove that no weak solution exists if

$$
N(p q-1)-2\left(p+1+k_{2}+k_{1} p\right)+\sigma_{2}+p \sigma_{1}<0 .
$$

The case when

$$
\begin{aligned}
& \min \left\{N(p q-1)-2\left(q+1+k_{1}+k_{2} q\right)+\sigma_{1}+q \sigma_{2},\right. \\
&\left.N(p q-1)-2\left(p+1+k_{2}+k_{1} p\right)+\sigma_{2}+p \sigma_{1}\right\}=0
\end{aligned}
$$

can be treated as in the proof of Theorem 2.4. This concludes the proof.

Remark 4.3. The nonexistence condition given in Theorem 4.2 includes all the parameters $k_{i}$ and $\sigma_{i}(i=1,2)$ and reduces to those found in [8,9] in the particular cases $k_{i}=0$ and $\sigma_{i}=0$, respectively. This solves a question posed in Deng and Levine [1]. 
284 Existence and nonexistence of global solutions

\section{Acknowledgement}

This work was partially supported by the Italian Ministero della Università e della Ricerca Scientifica e Tecnologica, funds $60 \%$ and $40 \%$.

\section{References}

[1] K. Deng and H. A. Levine, The role of critical exponents in blow-up theorems: the sequel, J. Math. Anal. Appl. 243 (2000), no. 1, 85-126. MR 2001b:35031. Zbl 942.35025.

[2] M. Escobedo and M. A. Herrero, Boundedness and blow up for a semilinear reactiondiffusion system, J. Differential Equations 89 (1991), no. 1, 176-202. MR 91j:35040. Zbl 735.35013.

[3] J. Giacomoni, Some results about blow-up and global existence to a semilinear degenerate heat equation, Rev. Mat. Complut. 11 (1998), no. 2, 325-351. MR 99j:35114. Zbl 922.35081.

[4] H. A. Levine, The role of critical exponents in blowup theorems, SIAM Rev. 32 (1990), no. 2, 262-288. MR 91j:35135.

[5] E. Mitidieri and I. Pohozaev, Nonexistence of weak solutions for some degenerate elliptic and parabolic problems on $\mathbf{R}^{N}$, to appear.

[6] _ Nonexistence of positive solutions for quasi-linear elliptic problems on $\mathbf{R}^{N}$, Proc. Steklov Inst. Math. 227 (1999), 186-216.

[7] _ Nonexistence of weak solutions for degenerate and singular hyperbolic problems on $\mathbf{R}^{N}$, Proc. Steklov Inst. Math. 232 (2000), 1-19.

[8] K. Mochizuki and Q. Huang, Existence and behavior of solutions for a weakly coupled system of reaction-diffusion equations, Methods Appl. Anal. 5 (1998), no. 2, 109-124. MR 99e:35109. Zbl 913.35065.

[9] Y. Uda, The critical exponent for a weakly coupled system of the generalized Fujita type reaction-diffusion equations, Z. Angew. Math. Phys. 46 (1995), no. 3, 366-383. MR 96m:35168. Zbl 835.35071.

Gabriella Caristi: Dipartimento di Scienze Matematiche Università di Trieste, I-34100 Trieste, ITALY

E-mail address: caristi@univ.trieste.it 


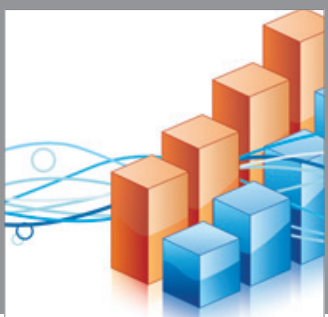

Advances in

Operations Research

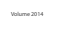

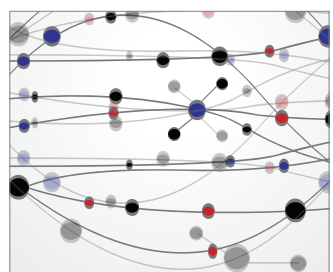

\section{The Scientific} World Journal
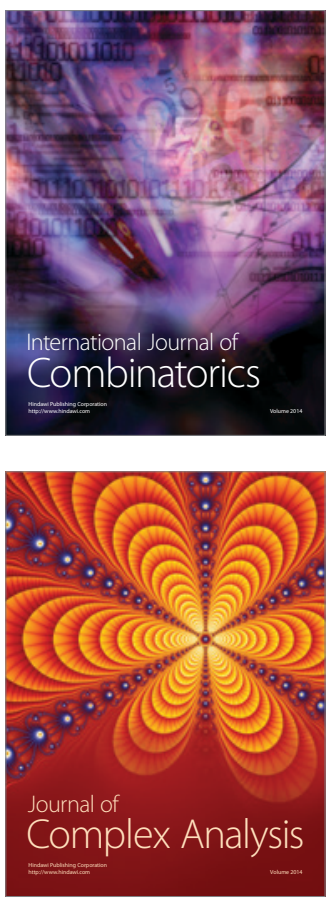

International Journal of

Mathematics and

Mathematical

Sciences
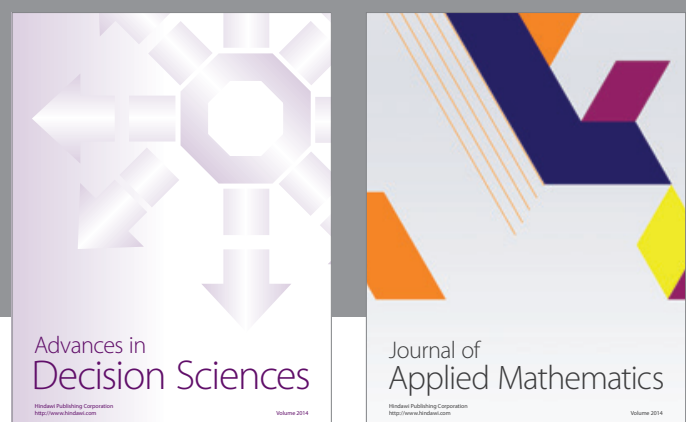

Journal of

Applied Mathematics
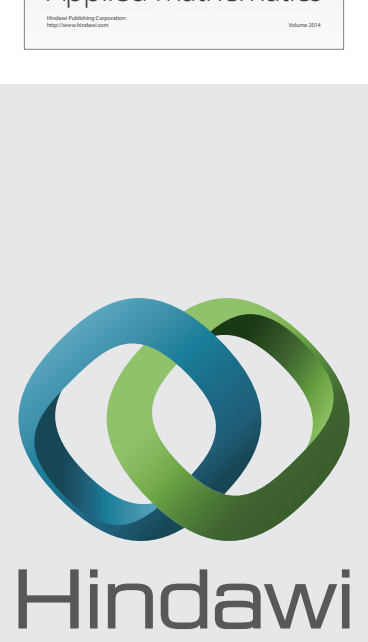

Submit your manuscripts at http://www.hindawi.com
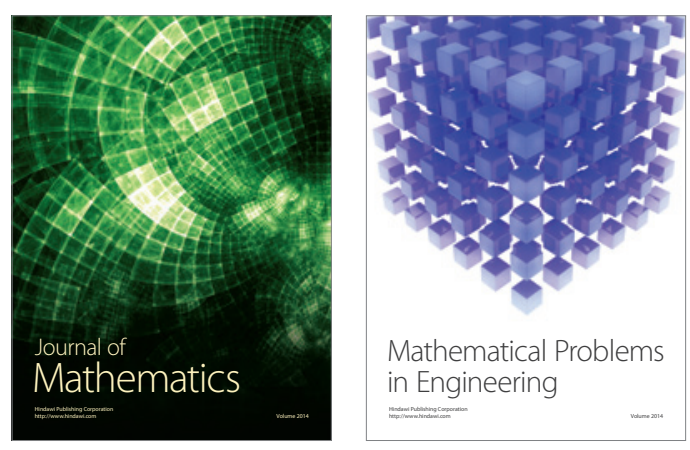

Mathematical Problems in Engineering
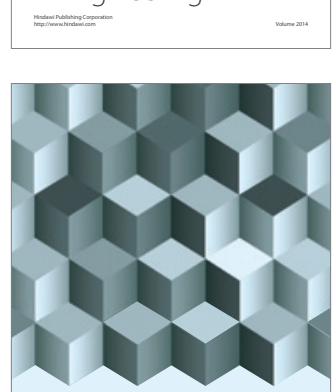

Journal of

Function Spaces
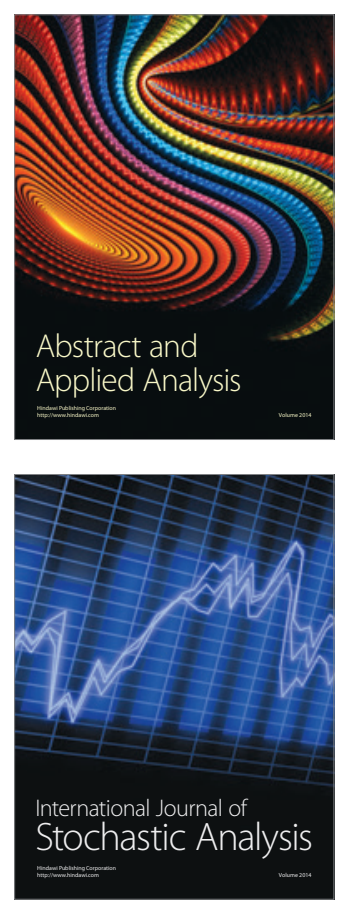

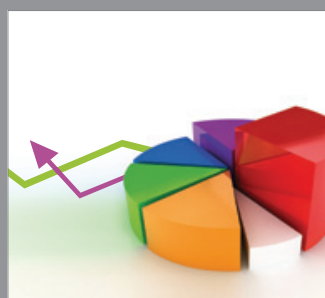

ournal of

Probability and Statistics

Promensencen
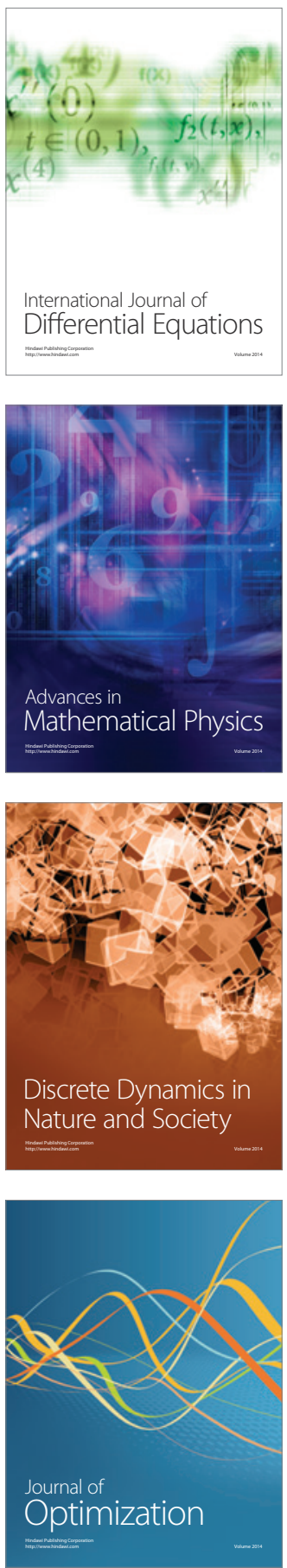